\title{
Benchmarking cataract surgery outcomes in an ethnically diverse and diabetic population: final post-operative visual acuity and rates of post-operative cystoid macular oedema
}

'Ophthalmology

Department, Whipps Cross University hospital, Leytonstone, London, UK

${ }^{2}$ Ophthalmology Department, Whipps Cross University Hospital, London, UK

${ }^{3}$ Medical Retina Department, Moorfields Eye Hospital, London, UK

${ }^{4}$ Ophthalmology Department, Whipps Cross University Hospital, London, UK

Correspondence:

K Oyewole, Ophthalmology Department, Whipps Cross University Hospital, Whipps Cross Road Leytonstone, London, EN11 1NR, UK Tel: +447908625128; Fax: +442085356466 E-mail: koyewole4@gmail. com

Received: 5 August 2016 Accepted in revised form: 16 April 2017

Published online: 23 June 2017

\begin{abstract}
Purpose To determine visual acuity and rates of post-operative cystoid macular oedema (CMO) in an ethnically diverse and predominantly diabetic population.

Methods The study was undertaken over a one year period. Snellen visual acuity (VA) was measured pre and 4-8 weeks postoperatively and optical coherence topography (OCT) was performed at baseline and postoperatively. No eyes received prophylactic non-steroidal anti-inflammatory drugs (NSAIDs) prior to or after surgery. Results Out of 262 eyes, $59 \%$ were in the Black, Asian and minority ethnic group (BAME), $57 \%$ had a history of diabetes mellitus and $34 \%$ had pre-existing diabetic retinopathy. $76 \%$ of all eyes achieved $6 / 12$ post-operative VA at the first postoperative review and the incidence of post-operative CMO within the study population was $7.6 \%$. In patients with a history of diabetes mellitus the incidence of post-operative CMO was $10.7 \%$ compared to $3.5 \%$ in those without diabetes. This was found to be clinically significant $(P=0.0297)$.

Conclusion We believe this is the first study to benchmark standards for care in this demographic of patients. A history of diabetes mellitus, irrespective of whether there is diabetic retinopathy or not, is an independent risk factor for the development of post-operative $\mathrm{CMO}$ in patients undergoing cataract surgery $(P=0.0297)$. Ethnicity is not an independent risk factor for the development of post-operative CMO.
\end{abstract}

K Oyewole ${ }^{1}$, F Tsogkas ${ }^{2}$, M Westcott ${ }^{3}$

and S Patra ${ }^{4}$
Eye (2017) 31, 1672-1677; doi:10.1038/eye.2017.96; published online 23 June 2017

\section{Introduction}

The Royal College of Ophthalmologists National Ophthalmology Database Audit (RCOphth NOD Audit) aims to analyse a nationally agreed cataract surgery data set and update the benchmark standards of care originally published through the Cataract National Database (CND) reports. ${ }^{1}$ However, both the CND and RCOphth NOD audit populations had much lower percentages of eyes with preexisting diabetic retinopathy undergoing cataract surgery than our study population. ${ }^{1,2}$ The study population of the CND was also ethnically less diverse with only $2.6 \%$ from the Black, Asian and minority ethnic group (BAME).

This difference in baseline characteristics of patients undergoing cataract surgery is important for several reasons. Diabetic retinopathy is a known risk indicator for worse visual acuity outcomes following cataract surgery. ${ }^{3}$ The Diabetic Retinopathy In Various Ethnic groups in UK (DRIVE UK) Study found a higher prevalence of both diabetic retinopathy (DR) and sight threatening DR in minority ethnic groups (South Asian, African and AfroCaribbean). ${ }^{4}$ In addition, post-operative cystoid macular oedema (CMO), associated with worse visual outcomes, occurs more frequently in eyes with diabetic retinopathy. ${ }^{5}$ The published rates for post-operative CMO from the CND analysis of 16731 cataract operations was 1.62 vs $16.7 \%$ 
reported in a study of eyes with pre-existing diabetic retinopathy (3 and 19). We could find no published data comparing post-cataract surgery CMO rates in BAME vs white ethnic groups.

Given that our study population had a higher percentage of patients with diabetes mellitus and a greater BAME group than the published audits, we postulated that the incidence of post-cataract surgery $\mathrm{CMO}$ would be higher in our study population. Therefore the final post-operative visual acuity would be worse than those published in the CND and RCOphth NOD reports. As secondary outcome we wanted to determine whether ethnicity was a contributory factor in the development of post-operative CMO.

\section{Materials and methods}

\section{Study population}

The study was undertaken in a busy inner city eye unit in London. The catchment area included the London boroughs of Waltham Forest, Redbridge, and Newham. The patients were recruited exclusively from the Retina firm and were under the care of a single Consultant surgeon. The cataract surgery was performed either by a Consultant or a senior trainee. Cases complicated by posterior capsule rupture (PCR) were excluded as it is a known risk factor for $\mathrm{CMO}$ and likely to confound the results. No eyes received pre or post-operative prophylaxis with non-steroidal anti-inflammatory drugs (NSAIDs).

\section{Outcome measures}

The primary outcomes analysed in this study population were: (a) the percentage of eyes achieving a final postoperative Snellen acuity of $6 / 12$ or better and (b) the percentage of eyes developing $\mathrm{CMO}$ following cataract surgery. The secondary outcomes included visual acuity outcomes and rates of post-operative CMO in the study sub-groups and statistical analysis of potential risk factors for post-operative CMO.

\section{Data collection}

The data was extracted from a single electronic medical records database (Medisoft Ophthalmology, Medisoft Ltd, Leeds, UK) over a 12 month period. The pre and post-operative Snellen visual acuity, ethnicity, diabetic status, grade of diabetic retinopathy, progression of diabetic maculopathy or retinopathy, development, treatment and outcome of post-operative $\mathrm{CMO}$ were recorded. The best recorded and pinhole visual acuity scores were used for both pre and post-operative vision.
The optical coherence tomography (OCT) measurements were recorded from the macular volume scans taken on the Zeiss Cirrus OCT machine.

\section{Diagnosis of post-operative CMO}

A baseline macular OCT scan was performed preoperatively in all eyes if the patient had a history of diabetes mellitus or there was pre-existing maculopathy of any aetiology such a retinal vascular disease or AMD.

In non-diabetic patients and if the eye to be operated was clinically normal on slit-lamp examination with a Volk Digital High Mag lens then a macular volume OCT scan was not routinely performed. All eyes had a macular volume OCT scan performed 4-8 weeks post-operatively to aid the diagnosis of post-operative $\mathrm{CMO}$ and differentiate between post-operative $\mathrm{CMO}$ and progression of pre-existing macular pathology. Postoperative $\mathrm{CMO}$ was diagnosed if the post-operative OCT scan showed typical features of intraretinal cysts and retinal thickening in an eye with either a normal macula on the pre-operative macular OCT scan or on slit-lamp examination with a Volk Digital High Mag lens. In eyes with abnormal pre-operative macular volume OCT scans, fundus fluorescein angiography was used to make the diagnosis.

\section{Statistics}

Statistical analysis was performed by using software SPSS ver (IBM SPSS Statistics for Windows, Version 19.0, IBM Corp, Armonk, NY, USA). Descriptive statistics including percentages and mean values were calculated for each group. Univariate analysis (two-sided $\chi^{2}$-test, or where appropriate Fischer' s exact test) was performed to identify factors associated with post-operative CMO in different groups. A $P$-value $<0.05$ was considered statistically significant. Due to the relatively small sample size the power to detect a difference was small, and so a type 2 error cannot be excluded.

\section{Results}

\section{Study demographics}

The data of 282 eyes of 245 patients was extracted over a 12 month period. Of these 20 eyes were excluded as 16 did not attend their post-operative follow-up, 2 had incomplete records and 2 had posterior capsule ruptures (PCR). The total number of eyes included in the results analysis was 262. The mean age was 71 years (range 39-95) and the male to female ratio was 2:3. The ratio of Consultant to senior trainee operated eyes was 2:1. 


\section{Ethnicity}

The study population was ethnically diverse with $40 \%$ (105/262) Asian, 18\% (47/262 ) African or Caribbean, 35\% (93/262) Caucasian and 6\% classified as undefined. The Black, Asian, and minority ethnic group (BAME) was 59\% accounting for over half the study population.

\section{Diabetic status}

The study population was predominantly diabetic with $57 \%(149 / 262)$ having a diagnosis of diabetes mellitus and $34 \%(88 / 262)$ having diabetic retinopathy. In the subgroup of the 149 eyes with a history of diabetes mellitus, 26 eyes had confirmed pre-operative diabetic macular oedema (DMO).

\section{Baseline visual acuity}

The pre-operative baseline Snellen visual acuity was $6 / 12$ or better in $26.3 \%$ of eyes and worse than $6 / 60$ in $6.5 \%$ of eyes.

\section{Management of the pre-existing maculopathy in eyes undergoing cataract surgery}

Of the 26 eyes that had pre-operative macular oedema, 15 were given 1-2 mg of intra-operative triamcinolone by intravitreal injection and 2 were given $0.5 \mathrm{mg}$ of intraoperative ranibizumab by intravitreal injection. The remaining nine eyes did not receive any intravitreal therapy as they were outside the treatment guidelines or there were contraindications such as glaucoma. The 17 eyes that received intra-operative intravitreal triamcinolone or ranibizumab to manage pre-existing maculopathy were excluded from the statistical analysis.

\section{Management of post-operative CMO in eyes undergoing cataract surgery}

No eyes received pre or post-operative prophylaxis with non-steroidal anti-inflammatory drugs (NSAIDs). Of the 20 eyes that developed OCT confirmed post-operative CMO, 19 were treated with a 6-8 week course of Acular eye drops three times a day to the affected eye combined with dexamethasone $0.1 \%$ eye drops four times a day to the affected eye. One eye was treated with post-operative intravitreal triamcinolone.

\section{Final post-operative visual acuity outcomes}

In the total study population the number of eyes that achieved $6 / 12$ or better Snellen acuity at the first post- operative review between 4-8 weeks was 76\% (198/262). This increased to $79 \%(207 / 262)$ after adjusting for the improved VA scores achieved following completion of treatment for the eyes that had developed post-operative CMO. Within the study population subgroups, patients with no history of diabetes mellitus had better visual acuity outcomes with $84 \%$ achieving a post operative visual acuity of $6 / 12$ or better, but this figure dropped to $68 \%$ in patients with diabetic retinopathy. Four eyes $(1.5 \%)$, found to have post-operative visual acuity $6 / 60$ or worse, had a guarded visual prognosis due to pre-existing co-morbidities and there was no deterioration in VA despite the surgical intervention. Five eyes (1.9\%) lost three or more lines of Snellen visual acuity following surgery. All these eyes had underlying pathologies which included pre-existing DMO, age-related macular degeneration, uveitis and refractive error in an amblyopic eye. Of the 20 eyes that developed post-operative CMO only two failed to achieve a final post-operative visual acuity of $6 / 12$ or better despite treatment. One of the two eyes with a poor visual outcome was later diagnosed as having $\mathrm{CMO}$ secondary to chronic uveitis rather than post-operative CMO.

\section{Incidence of post-operative CMO}

The incidence of post-operative CMO within the study population was 20 eyes out of 262 (7.6\%). This does not include 17 eyes with a pre-existing macular oedema secondary to either diabetes (14 eyes) or retinal vein occlusion (3 eyes) that had been treated intra- operatively with either triamcinolone or ranibizumab. In eyes without a history of diabetes mellitus the incidence of postoperative $\mathrm{CMO}$ was 3.5\% and in eyes with a history of diabetes mellitus the incidence of post-operative CMO was $10.7 \%$. This difference was found to be clinically significant $(P=0.0297)$. The post-operative $\mathrm{CMO}$ rate by surgeon grade was $6.3 \%$ for Consultants and $10 \%$ for senior trainees but this difference did not reach statistical significance. The rate of post-operative $\mathrm{CMO}$ by ethnic group was $10.5 \%$ in the BAME group vs $2.6 \%$ in the white ethnic group. The high prevalence of diabetes mellitus in the BAME ethnic group was found to be a confounding factor, which when adjusted for revealed that ethnicity was not an independent risk factor for post-operative $\mathrm{CMO}$ in our study population.

\section{Discussion}

We report the post-operative visual acuity and incidence of post-operative CMO following uncomplicated phacoemulsification cataract extraction and lens 
Table 1 This table compares key differences in the baseline characteristics and outcomes between our study population the Royal College of Ophthalmologists NOD audit and the Cataract National Database audit

\begin{tabular}{lccc}
\hline & Study cohort all eyes (\%) & RCOphth NOD audit all eyes (\%) & CND audit all eyes (\%) \\
\hline Baseline VA equal to or better than 6/12 & 26.3 & 32 (first eye) 47.7 (second eye) & 42.9 \\
Diabetic retinopathy & 34 & 4.7 & 3.3 \\
Post-op VA equal to or better 6/12 & 79 & 89.9 & 91 \\
Post op CMO & 7.6 & Not reported & 1.62 \\
\hline
\end{tabular}

implantation in an urban, predominately Black, Asian, and minority Ethnic (BAME) and diabetic population.

Within our study cohort $76 \%$ had a visual acuity (VA) equal to or better than $6 / 12$ post operatively increasing to $79 \%$ when including VA scores after completion of treatment for post operative CMO. This was lower than the reported VA outcomes for both the RCOphth NOD and the CND audit.

The overall incidence of post-operative CMO within the study group was $7.6 \%$. It was $10.7 \%$ in patients with a history or diabetes mellitus regardless of whether or not they had diabetic retinopathy and much lower, only 3.5\%, in patients with no history of diabetes mellitus. These rates were higher than the incidence of post-operative $\mathrm{CMO}$ reported in the CND audit.

One of the main reasons for these observed differences in VA outcomes and post-op CMO rates was postulated to be the wide difference in the baseline demographics of our study population as demonstrated in Table 1.

In our study population over half or $57 \%$ had a history of diabetes mellitus with $34 \%$ having diabetic retinopathy at the time of surgery, compared to the RCOphth NOD audit and CND audit of 4.7 and 3.3\% with diabetic retinopathy, respectively. Diabetic retinopathy is a known risk indicator for worse visual acuity outcomes following cataract surgery. ${ }^{3}$

In addition, within the RCOphth NOD audit $32 \%$ of first eyes and $47.7 \%$ of second eyes had a pre-operative VA equal to or better than $6 / 12$. Similarly $42.9 \%$ had a VA equal to or better than $6 / 12$ in the CND audit. Within our study population only $26.3 \%$ had a VA equal to or better than 6/12 pre-operatively. The Early treatment Diabetic Retinopathy Study (ETDRS) identified poor pre-operative VA as a risk factor for poor postoperative outcome, possibly attributed to macular oedema, macular ischaemia or traction. ${ }^{5}$ In addition, they reported that the severity of DR at the time of cataract surgery affected the post operative outcome suggesting that this could be associated with an increased incidence of macular ischaemia. $^{5}$

Our study population consisted of 59\% Black, Asian and minority ethnic groups compared to $2.6 \%$ in the CND audit. Although ethnicity was not found to be an independent risk factor for post operative CMO within our study, the Diabetic
Retinopathy In Various Ethnic groups in UK (DRIVE UK) study found a higher prevalence of both diabetic retinopathy (DR) and sight threatening DR in minority ethnic groups (South Asian, African, and AfroCaribbean). ${ }^{4}$ Diabetes has been associated with poorer outcomes post-cataract surgery. Post-operative inflammation is thought to be the most important factor in the development of postoperative complications within diabetic subjects. Menchini et al reported intraocular inflammation and its sequelae as the most common complication in their study. ${ }^{6}$ Similarly, Ivancic et al. reported that inflammatory reactions and bleeding were more common in diabetic patients postcataract surgery resulting in anterior segment complications including post-operative keratopathy, fibrinous uveitis and posterior capsule opacity. ${ }^{7}$ Greenberg et $a l^{8}$ showed that of 45082 patients undergoing cataract extraction, subjects with diabetic retinopathy had a 33\% higher operative complication risk compared with those without diabetic retinopathy. Furthermore several studies have shown that diabetic patients are at higher risk of, DMO worsening, ${ }^{9-11} \mathrm{DR}$ progression, ${ }^{12}$ and CMO. ${ }^{13}$

Risk factors for postoperative complications have been linked to the severity of retinopathy and maculopathy prior to cataract surgery, higher HbA1c levels, ${ }^{14}$ and type 2 diabetes. ${ }^{15}$ Our findings demonstrated that a history of diabetes mellitus in patients undergoing cataract surgery is an independent risk factor for the development of postoperative CMO $(P=0.0297)$.

This supports published data that macular changes are more likely to occur in diabetic patients following uncomplicated cataract surgery with incidences reported as ranging from 31 to $81 \% .{ }^{16-18}$ It is believed that release of prostaglandins in the postoperative inflammatory process causes fluid leakage from perifoveal capillaries into the extra cellular space within the retina. Muller cell lysis occurs secondary to excessive fluid accumulation with fluid collecting in the outer plexiform and inner nuclear layers of the retina. It is likely that diabetic patients are more susceptible to this postoperative inflammatory response due to an already altered blood retinal barrier secondary to hyperglycaemia and subclinical inflammation. 
Following treatment for $\mathrm{CMO}$ with topical keratolac and dexamethasone for 6-8 weeks, $90 \%$ eyes that developed post-operative $\mathrm{CMO}$ regained a VA of equal to or better than $6 / 12$ with resolution of $\mathrm{CMO}$ on the macular OCT scans. No prophylaxis therapy was used in the study cohort. However a recent study has clearly demonstrated that in diabetic patients with nonproliferative diabetic retinopathy, treatment with the NSAID Nepafenac $0.1 \%$ eye drops 1 day prior to surgery through to day 90 post operatively can significantly reduce the incidence of post-operative $\mathrm{CMO}$ from 16.7 to $3.2 \% .{ }^{19}$ A more recent meta-analysis showed that in diabetic patients with or without diabetic retinopathy the optimum treatment regime to reduce the incidence of post-operative $\mathrm{CMO}$ was a combination of topical dexamethasone and NSAID post operatively in diabetic patients. $^{20}$

It will be soon be mandatory for individual surgeons to publish cataract surgery outcomes. Given the differences in the demographic make up within different parts of the UK is it possible to compare such different population groups?

\section{Summary}

What was known before

- Diabetic retinopathy is a risk factor for worse postoperative visual acuity outcomes. The incidence of cystoid macular oedema following cataract surgery is higher in diabetic patients. Postoperative cystoid macular oedema more common in diabetic patients.

What this study adds

- We believe this is the first study to benchmark standards for care in this demographic of patients.

- A history of diabetes mellitus, irrespective of whether they have diabetic retinopathy or not, is an independent risk factor for the development of postoperative $\mathrm{CMO}$ in patients undergoing cataract surgery $(P=0.0297)$. Ethnicity is not an independent risk factor for the development of postoperative CMO. Postoperatively, cataract surgery cystoid macular oedema is more common in diabetic patients irrespective of whether the patient has diabetic retinopathy. Ethnicity is not a risk factor for post operative cystoid oedema.

\section{Conflict of interest}

The authors declare no conflict of interest.

\section{References}

1 Day AC, Donachie PH, Sparrow JM, Johnston RL. Royal College of Ophthalmologists' National Ophthalmology database. The Royal College of
Ophthalmologists, National Ophthalmology Database study of cataract surgery: report 1 , visual outcomes and complications. Eye 2015; 29: 552-560.

2 Jaycock P, Johnston RL, Taylor H, Adams M, Tole DM, Galloway $\mathrm{P}$ et al. The Cataract national dataset electronic multi-centre audit of 55567 operations: updating benchmark standards of care in the United Kingdom and Internationally. Eye 2009; 23: 38-49.

3 Sparrow JM, Taylor H, Qureshi K, Smith R, Birnie K, Johnston RL. The Cataract National Dataset electronic multi centre audit of 55567 operations: risk indicators for monocular visual acuity outcome. Eye 2012; 26: 821-826.

4 Sivaprasad S, Gupta B, Gulliford MC, Dodhia H, Mann S, Nagi D et al. Ethnic Variations in the Prevalence of Diabetic Retinopathy in People attending Screening in the United Kingdom (DRIVE UK). PLoS One 2012; 7(3): e32182.

5 Chew EY, Benson WE, Remaley NA, Lindley AA, Burton $\mathrm{TC}$, Csaky $\mathrm{K}$ et al. Results after lens extraction in patients with diabetic retinopathy: early treatment diabetic retinopathy study report number 25. Arch Ophthalmol 1999; 117(12): 1600-1606.

6 Mechini U, Cappelli S, Virgili G. Cataract surgery and diabetic retinopathy. Semin Ophthalmol 2003; 18: 103-108.

7 Ivancic D, Mandic Z, Barac C, Kopic M. Cataract surgery and post operative complication in diabetic patients. Coll Antropol 2005; 29(Suppl 1): 55-58.

8 Greenberg PB, Tseng VL, Wu WC, Liu J, Jiang L, Chen CK et al. Prevalence and predictors of ocular complications associated with cataract surgery in United States veterans. Ophthalmology 2011; 118(3): 507-514.

9 Kim SJ, Equi R, Bressler NM. Analysis of macuar oedema after cataract surgery in patients with diabetes using optical coherence tomography. Ophthalmology 2007; 114(5): 881-889.

10 Pate JL, Hykin PG, Cree IA. Diabetic cataract removal: Postoperative progression of maculopathy: Growth factor and clinical analysis. Br J Ophthalmology 2006; 90(6): 697-701.

11 Kim SJ, Belair ML, Bressler NM, Dunn JP, Thorne JE, Kedhar SR et al. A method of reporting macular oedema after cataract surgery using optical coherence tomography. Retina 2008; 28(6): 870-876.

12 Gupta A, Gupta V. Diabetic maculopathy and cataract surgery. Ophthalmol Clin North Am 2001; 14(4): 625-637.

13 Mechini, Bandello F, Brancato R, Camesasca FI, Galdini M. Cystoid macular oedema after extracapsular cataract extraction and lens implantation in diabetic patients without retinopathy. Br J Ophthalmol 1993; 77(4): 208-211.

14 Diabetes Control and Complications Trial Research Group. The Diabetes Control and Complications Trial. The effect of intensive diabetes treatment on the progression of diabetic retinopathy in insulin-dependent diabetes mellitus. Arch Ophthalmol 1995; 113(1): 36-51.

15 Flesner P, Sander B, Henning V, Parving HH, Dornonville de la Cour M, Lund-Andersen H. Cataract surgery on diabetic patients: a prospective evaluation of risk factors and complications. Acta Ophthalmol Scand 2002; 80(1): 19-24.

16 Tranos PG, Wickremasinghe SS, Stangos NT, Topouzis F, Tsinopoulos I, Pavesio CE. Macular oedema. Surv Ophthalmol 2004; 49: 470-490. 
17 Ursell PG, Spalton DJ, Whitcup SM, Nussenblatt RB. Cystoid macular oedema after phacoemulsification: relationship to blood-aqueous barrier damage and visual acuity. J Cataract Refract Surg 1999; 25: 1492-1497.

18 Hayashi K, Igarashi C, Hirata A, Hayashi H. Changes in diabetic macular oedema after phacoemulsification surgery. Eye (Lond) 2009; 23: 389-396.

19 Singh R, Alpern L, Jaffe GJ, Lehmann RP, Lim J, Reiser $\mathrm{HJ}$ et al. Evaulation of nepafenac in prevention of macular edema following cataract surgery in patients with diabetic retinopathy. Clin Ophthalmol 2012; 6: 1259-1269.

20 Wielders L, Lambermont VA, Schouten JS, van den Biggelaar FJ, Worthy G, Simons RW et al. Prevention of cystoid macular Edema after cataract surgery in nondiabetic and diabetic patients; a systemic review and meta-analysis. Am J Ophthalmol 2015; 160(5): 968-981. 